\title{
Anabases
}

ANABASES Traditions et réceptions de l'Antiquité

$21 \mid 2015$

Varia

\section{La correspondance d'Émile Espérandieu au Palais du Roure à Avignon}

Marianne Altit-Morvillez

\section{OpenEdition}

1 Journals

Édition électronique

URL : http://journals.openedition.org/anabases/5336

DOI : 10.4000/anabases.5336

ISSN : 2256-9421

Éditeur

E.R.A.S.M.E.

Édition imprimée

Date de publication : 1 avril 2015

Pagination : 247-252

ISSN : 1774-4296

Référence électronique

Marianne Altit-Morvillez, « La correspondance d'Émile Espérandieu au Palais du Roure à Avignon »,

Anabases [En ligne], 21 | 2015, mis en ligne le 01 avril 2018, consulté le 21 octobre 2019. URL : http:// journals.openedition.org/anabases/5336 ; DOI : 10.4000/anabases.5336

Ce document a été généré automatiquement le 21 octobre 2019

(c) Anabases 


\title{
La correspondance d'Émile Espérandieu au Palais du Roure à Avignon
}

\author{
Marianne Altit-Morvillez
}

1 Parmi les archéologues de la première moitié du $\mathrm{xx}^{\mathrm{e}}$ siècle, le commandant Émile Espérandieu est connu essentiellement pour son ouvrage le Recueil des Bas-Reliefs de la Gaule, mais une grande partie de son œuvre reste quelque peu occultée. Par ailleurs, sa double carrière de militaire et d'archéologue le rend inclassable, car il ne fait pas partie des institutions universitaires de son temps. En étudiant, pour ma thèse ${ }^{1}$, ses archives conservées au Palais du Roure à Avignon, et en particulier sa correspondance scientifique, j'ai voulu non seulement appréhender le parcours d'un militaire hors norme dans le contexte historiographique de la société archéologique de la III ${ }^{\mathrm{e}}$ République, mais aussi, à travers ce prisme, tenter de rendre compte de manière plus globale des interactions sociales et des pratiques scientifiques au sein des réseaux archéologiques métropolitains ${ }^{2}$.

2 Pour appréhender la richesse du fonds documentaire du Palais du Roure, je rappellerai d'abord sa carrière. Émile Espérandieu, né en 1857, est fils d'un agriculteur d'un petit village du Gard (Saint-Hippolyte-de-Caton). Pendant son service militaire, ses supérieurs le remarquent et sur leurs conseils, il passe le baccalauréat pour entrer en 1878 à l'école spéciale de Saint-Cyr. Envoyé à sa demande dans le Corps expéditionnaire de Tunisie, il est affecté aux brigades topographiques. Là, il découvre l'épigraphie et l'archéologie, comme beaucoup d'officiers dans les colonies d'Afrique ${ }^{3}$ et rencontre les érudits locaux, comme Alexandre Papier ${ }^{4}$, ou le Père Delattre ${ }^{5}$. Il commence une correspondance scientifique, et envoie ses premiers relevés d'épigraphie à l'Académie d'Hippone, et à l'Académie des Inscriptions et Belles-Lettres ${ }^{6}$.

Cette expérience scientifique va se développer lorsqu'il rentre en métropole en 1884, car il s'intéresse au gré de ses affectations à l'archéologie locale et intègre de nombreuses sociétés savantes. C'est pour lui une période féconde de publications de corpus d'épigraphie régionale qui le font remarquer du monde savant ${ }^{7}$. Il développe son 
réseau scientifique tant en France qu'à l'étranger : il devient le disciple et l'ami d'Auguste Allmer ${ }^{8}$, fondateur de la Revue épigraphique du Midi de la France, publication dont il prendra la direction en 1898 ; il sollicite et reçoit les conseils de R. Cagnat ${ }^{9}$ qu'il $^{\prime}$ avait rencontré en Tunisie $^{10}$, de S. Reinach ${ }^{11}$, ou d'A. Héron de Villefosse ${ }^{12}$; il entre en contact avec les éditeurs du cIL, O. Hirschfeld ${ }^{13}, \mathrm{O}$. Bohn ${ }^{14}$, leur envoie ses inscriptions de Tunisie, et en 1904, publiera dans le tome XIII son Recueil des cachets d'oculistes romains ${ }^{15}$.

Dans le même temps, professeur à l'école des officiers de Saint-Maixent, il publie un cours de géographie, un autre de topographie ; il est chargé aussi, par sa hiérarchie qui profite ainsi de ses compétences, de recherches d'histoire militaire ${ }^{16}$. À partir de 1901, détaché à la direction de la Revue du Cercle militaire - à cause d'une surdité croissante, il ne peut plus enseigner -, il s'installe à Paris et est nommé correspondant de l'Institut. En 1903, le ministère de l'Instruction publique le charge, sur la recommandation de C. Jullian ${ }^{17}$ et $\mathrm{S}$. Reinach, de la préparation du Recueil général des bas-reliefs de la Gaule romaine. Il est ensuite nommé à la direction des fouilles d'Alésia en 1905. Chaque année, il publie les rapports de fouilles et travaille au Recueil des bas-reliefs. Lorsque la guerre éclate en 1914, alors qu'il était en retraite depuis l'année précédente, il reprend volontairement un service de bureau à l'armée, le front lui étant refusé à cause de son handicap. En 1919, il est élu à l'Académie des Inscriptions et Belles-Lettres et nommé conservateur des musées de Nîmes la même année. Il reprend ses fouilles d'Alésia, et poursuit la publication des Bas-reliefs, dont le dernier tome $\mathrm{XI}^{18}$ est publié en 1938, un an avant sa mort.

5 Cette carrière longue et complexe explique la variété des documents du Palais du Roure : sont conservés, par la volonté même d'Espérandieu, non seulement l'intégralité de sa bibliothèque, mais aussi ses manuscrits, sa documentation iconographique, et sa correspondance. Celle-ci n'était pas accessible à la recherche car elle n'avait jamais été classée entièrement. Pour étudier cette correspondance scientifique ${ }^{19}$, il m'a donc fallu la classer dans un premier temps, et l'inventorier. En l'état actuel de mon inventaire, qui n'est pas définitif, les correspondants sont près de 2500 , pour environ 15000 lettres. Le nombre des correspondants semble très important - d'autant que l'archéologie constitue la deuxième carrière du commandant -, mais n'est pas exceptionnel au regard d'autres correspondances d'archéologues inventoriées, comme celles de F. Cumont ${ }^{20}$, ou de J. Déchelette ${ }^{21}$. Cette correspondance passive trouve un intérêt augmenté par des lettres de la correspondance active retrouvée dans des fonds accessibles $^{22}$ et complétée par le document autobiographique des Souvenirs rédigés entre 1931 et 1936. Ainsi un choix de 195 lettres et les Souvenirs ont été retranscrits en appui de la synthèse de mon travail.

6 J'ai abordé cette correspondance pléthorique à travers trois thématiques clés de la carrière d'Espérandieu : l'épigraphie, l'édition du Recueil des bas-reliefs, et la pratique de l'archéologie qui ont permis de relier les problématiques de manière transversale. En premier lieu, analyser les correspondances permet de comprendre la position de son statut d'officier saint-cyrien et son évolution de l'érudit amateur à l'expert reconnu. Le parcours met en valeur son ascension sociale : Espérandieu est l'exemple type du système de la III ${ }^{e}$ République de l'élévation par le mérite. Ainsi, la correspondance montre bien le fonctionnement d'un cursus honorum, depuis la reconnaissance institutionnelle des travaux scientifiques pour les érudits provinciaux, jusqu'à l'entrée à l'Académie des Inscriptions et Belles-Lettres, dont Espérandieu est l'exemple théorique mais exceptionnel en pratique ${ }^{23}$. 
7 Par ailleurs, on comprend la stratégie mise en place par Espérandieu pour s'intégrer dans le monde érudit des sociétés savantes ${ }^{24}$, bien loin de l'armée, son cadre professionnel d'origine.

8 Les échanges nourris avec les réseaux, et avec les institutionnels de Paris, permettent de saisir leurs imbrications. C'est ainsi que le traitement de l'inventaire a mis en exergue le maillage du réseau : on retrouve la plupart des savants et des sociétés savantes de l'époque s'occupant d'archéologie gallo-romaine. Certains comme S. Reinach, C.Jullian, Héron de Villefosse se distinguent par leur volume d'échanges marquant de facto leur importance non seulement dans l'évolution de la carrière d'Espérandieu, mais aussi au sein de ces réseaux scientifiques. Parmi les correspondants étrangers, les Allemands sont, sans surprise, les plus nombreux puisqu'Espérandieu a participé au cIL, et édité en grande partie grâce à leur aide les basreliefs du limes.

9 Au-delà de ce portrait, cette correspondance nous donne à voir les interactions sociales des réseaux. Les sociétés savantes montrent, en même temps, une grande disponibilité dans les échanges scientifiques, et dans l'accueil des membres, mais aussi une volonté forte de garder leur autonomie, en particulier vis-à-vis de Paris : Espérandieu en fait l'expérience sur le chantier d'Alésia, avec le différend qui l'oppose à la Société de Semur. D'autre part, le rôle des sociétés de province dans la construction des savoirs transparaît indéniablement dans cette correspondance. D'un côté, on remarque l'indépendance d'un grand nombre de travaux antiquaires qui se font au sein des sociétés savantes de province. D'un autre côté, la supervision de ces travaux et leur soutien, guidage, critique par les institutions parisiennes, en particulier le cTHS, sont effectifs. En particulier, la correspondance montre que S. Reinach, C. Jullian suivent de très près l'avancée du Recueil des bas-reliefs. De même, apparaît le rôle de production mais aussi de passeur des sociétés savantes dans la recherche archéologique. Le maillage de ces sociétés sur tout le territoire et le système de diffusion par les bulletins des sociétés savantes sont fondamentaux. Dans cette optique, apparaît la question de la pratique éditoriale avec les problèmes de diffusion liés à la vitalité des réseaux, et de concurrence interne. Ce phénomène a pu être analysé à travers l'exemple de la Revue épigraphique.

10 Les archives permettent donc de dresser un portrait de ce militaire-archéologue - ainsi qu'Espérandieu se nomme lui-même -, mais, de manière concomitante, de nuancer fortement le rôle de l'armée dans l'histoire de l'archéologie. Si Espérandieu par son parcours original a pu faire une carrière savante, c'est tout à fait exceptionnel pour un officier sur le sol métropolitain, encore plus que dans les colonies ${ }^{25}$. Lorsqu'on replace dans son contexte la position de l'institution militaire, on constate qu'elle n'apprécie pas ces occupations non militaires. Toutefois, dans le cas d'Espérandieu, elle reste ambiguë : on la voit freiner la carrière d'Espérandieu mais se servir de ses compétences pour lui commander des travaux d'histoire militaire. Sans doute pourra-t-on affiner les relations de l'armée avec l'archéologie en étudiant la correspondance militaire encore inédite.

11 Enfin, ce qui apparaît remarquable dans le parcours d'Espérandieu, c'est sans doute sa position d'indépendant : membre de plusieurs sociétés savantes, il n'est jamais vraiment ancré dans un territoire, puisque, en tant que militaire, il ne reste pas longtemps en poste dans une même ville. C'est bien par et grâce à la correspondance qu'il maintient et agrandit ses réseaux. Son indépendance lui permet d'avoir les liens 
sans concurrence avec de nombreux archéologues, comme J. Déchelette ${ }^{26}$. Il faut noter la qualité de ses relations avec ses homologues allemands, surtout après la guerre. Alors que les académies européennes ont coupé les relations avec les académies allemandes ${ }^{27}$, il continue, bien qu'académicien lui-même, à travailler avec eux pour le Recueil des basreliefs, les lettres en témoignent.

Mais a contrario, si cette indépendance sert la carrière d'Espérandieu, d'une certaine manière, à cause d'elle, il reste à la marge. Il est symptomatique de constater que pour s'ancrer dans ce monde érudit, ce militaire se crée avec son maître en épigraphie Auguste Allmer une généalogie scientifique issue de l'héritage antiquaire ${ }^{28}$. Et sans doute du fait de son indépendance et de son statut militaire, il restera aussi toujours à la frontière entre l'antiquaire et l'archéologue, autre marge, celle-là scientifique et non plus institutionnelle ${ }^{29}$.

13 L'inventaire informatique de la correspondance d'Espérandieu, que je réalise actuellement à la demande de S. Clap, le conservateur du Palais du Roure, permettra d'apporter, à terme, de nouvelles données accessibles pour les recherches en cours sur l'histoire de l'archéologie gallo-romaine de la III République.

\section{NOTES}

1. Émile Espérandieu (1857-1939), un archéologue entre institution militaire et monde académique, Université Paris I, sous la direction d'Alain Schnapp, soutenue en septembre 2014.

2. Pour les enjeux de la recherche, C. BONNET, V. KRINGS (ÉD.), S'ÉCRIRE ET ÉCRIRE SUR L'ANTIQUITÉ. L'APPORT DES CORRESPONDANCES À L'HISTOIRE DES TRAVAUX SCIENTIFIQUES, JÉRÔME MILLON, GRENOBLE, 2008. L'HISTOIRE DES GRANDES INSTITUTIONS ARCHÉOLOGIQUES A ÉTÉ DÉVELOPPÉE DEPUIS DE NOMBREUSES ANNÉES, CF. E. GRAN-AYMERICH, LA NAISSANCE DE L'ARCHÉOLOGIE MODERNE (1798-1945), CNRS ÉDITIONS, PARIS, 1998. EN REVANCHE L'ARCHÉOLOGIE MÉTROPOLITAINE, À CAUSE DE SA SITUATION SPÉCIFIQUE, A FAIT L'OBJET D’ÉTUDES FORT DISPERSÉES, COMME L'EXPLIQUE O. PARSIS-BARUBÉ, LA PROVINCE ANTIQUAIRE. L'INVENTION DE L'HISTOIRE LOCALE EN FRANCE (1800-1870), CTHS, PARIS, 2011, P. 297.

3. M. KHANOUSSI, P. RUgGerI, C. VISMARA (DIR.), « L'AFRICA ROMANA. GEOGRAFI, VIAGGIATORI, MILITARI NEL MAGHREB : ALLE ORIGINI DELL'ARCHEOLOGIA NEL NORD AFRICA », L'AFRICA ROMANA 13, ROMA, 2000.

4. Alexandre Papier (1826-1909), président de l'Académie d'Hippone.

5. Alfred-Louis Delattre (1850-1932), prêtre de la Société des Missionnaires d'Afrique (Pères blancs), archiprêtre de la Primatiale de Carthage, conservateur du Musée Lavigerie de Saint-Louis de Carthage.

6. Ces inscriptions seront regroupées dans Épigraphie des environs du Kef, Paris 1884-1885, en 7 fascicules.

7. Parmi ses publications, citons : Épigraphie romaine du Poitou et de la Saintonge, Melle, 1888 ; Inscriptions de la cité des Lemovices, Thorin, Paris, 1891 ; Inscriptions antiques de Lectoure, Paris, 1892 ; Inscriptions antiques de la Corse, Bastia, 1893.

8. Auguste Allmer (1815-1899), épigraphiste, conservateur du musée de Lyon, correspondant de l'Institut, son volume publié avec P. DISSARD, INSCRIPTIONS ANTIQUES DU MUSÉE DE LYON, LYON, 1888-1893, REÇOIT LE PRIX GOBERT. 
9. René Cagnat (1852-1937), professeur au Collège de France, sur la chaire d'épigraphie latine, fondateur de L'Année épigraphique, membre de l'Académie des Inscriptions et Belles-Lettres.

10. F. BARATTE, LE VOYAGE EN TUNISIE DE CAGNAT ET SALADIN, ÉDITÉ ET PRÉSENTÉ PAR F. BARATTE, CTHS, PARIS, 2005.

11. Salomon Reinach (1858-1932), archéologue, philologue, conservateur du musée des Antiquités nationales à Saint-Germain-en-Laye, professeur d'histoire de l'art et d'archéologie nationale à l'École du Louvre, membre de l'Académie des Inscriptions et Belles-Lettres.

12. Antoine Héron de Villefosse (1845-1919), conservateur au département des Antiquités grecques et romaines du Louvre, enseigne l'épigraphie à l'École pratique des hautes études, membre de l'Académie des Inscriptions et Belles-Lettres.

13. Otto Hirschfeld (1843-1922), épigraphiste, professeur d'histoire ancienne, succède à Mommsen à la direction du CIL.

14. Oscar Bohn (1857-1927), épigraphiste et collaborateur du CIL XIII.

15. Paru d'abord dans la Revue archéologique en 1893.

16. Expédition de Sardaigne et Campagne de Corse, 1793-1794, Paris, 1895 ; Histoire abrégée des Campagnes du $61^{e}$ Régiment d'Infanterie, d'après les archives du ministère de la Guerre, Marseille, 1897.

17. Camille Jullian (1859-1933), historien, philologue, professeur d'histoire et d'antiquités nationales à la faculté de Bordeaux, puis au Collège de France, membre de l'Académie des Inscriptions et Belles-Lettres et de l'Académie française.

18. Le recueil est complété par R. LANTIER, RECUEIL DES BAS-RELIEFS, T. XII-XV, 1947-1966, ET POUR LES TABLES DES NOTICES ET DES NOMS GÉOGRAPHIQUES, P.-M. DUVAL, T. XVI, 1981. IL EST REMIS À JOUR DEPUIS 2003, SOUS LA DIRECTION D’H. LAVAGNE, SOUS LE TITRE DE NOUVEL ESPÉRANDIEU, RECUEIL GÉNÉRAL DES SCULPTURES SUR PIERRE DE LA GAULE.

19. La conservatrice S. Barnicaud (1949-2011) m'a donné libéralement accès à cette correspondance scientifique. Notons que les correspondances militaires et privées ne sont ni classées, ni inventoriées.

20. En ligne sur le site de l'Academia Belgica :

http://www.academiabelgica.it/index.php?

option=com_content\&view=article\&id=113\&Itemid=90\&lang=fr

21. En ligne sur le site de la bibliothèque de Roanne : http://roanne.cd-script.fr/fr/listefond.php?id=7

22. Fonds Reinach au musée des Antiquités nationales de Saint-Germain-en-Laye et à la bibliothèque Méjanes à Aix-en-Provence, fonds Delattre aux Archives générales des missionnaires d'Afrique à Rome, Archives Gallia, Maison de l'Archéologie René-Ginouvès à Nanterre.

23. M. ALTIT-MORVILLEZ, S. PÉRÉ-NOGUES, « LE LONG CHEMIN VERS L'INSTITUT », IN REGARDS SUR L'ARCHÉOLOGIE, ACTES DES JOURNÉES D’ÉTUDES, 4-5 NOV. 2011, RECUEIL DE MÉMOIRES ET DOCUMENTS SUR LE FOREZ, LA DIANA, FRAL, T. 48, 2013, P. 39-48.

24. J.-P. CHALINE, SOCIABILITÉ ET ÉRUDITION. LES SOCIÉTÉS SAVANTES EN FRANCE, XIX E-XXE SIÈCLES, CTHS, PARIS, 1998.

25. M. DONDIN-PAYRE, « L'EXERCITUS AFRICAE INSPIRATRICE DE L'ARMÉE FRANÇAISE D'AFRIQUE : ENSE ET ARATRO », ANTIQUITÉS AFRICAINES, 27, 1991, P. 141-149.

26. Joseph Déchelette (1862-1914), industriel, archéologue protohistorien, auteur du Manuel d'archéologie préhistorique, celtique et gallo-romaine, fouilleur de Bibracte, conservateur du musée de Roanne, correspondant de l'Institut. M. ALTIT-MORVILLEZ, «LA CORRESPONDANCE ESPÉRANDIEUDÉCHELETTE RECONSTITUÉE », ANABASES, 9, 2009, P. 221-238.

27. R. REINBOTHE, « L'EXCLUSION DES SCIENTIFIQUES ALLEMANDS ET DE LA LANGUE ALLEMANDE DES CONGRÈS SCIENTIFIQUES INTERNATIONAUX APRÈS LA PREMIÈRE GUERRE MONDIALE », LA FABRIQUE INTERNATIONALE DE LA SCIENCE, REVUE GERMANIQUE INTERNATIONALE 12, 2010, P. 193-208 ; C. BONNET, LE « GRAND ATELIER DE LA SCIENCE ». FRANZ CUMONT ET L'ALTERTUMSWISSENSCHAFT. HÉRITAGES ET ÉMANCIPATIONS, INSTITUT HISTORIQUE BELGE DE ROME, ÉTUDES DE PHILOLOGIE, D'ARCHÉOLOGIE ET D'HISTOIRE ANCIENNES, 41, BRUXELLES-ROME, 2005, P. 355-375. 
28. F. WAQUET, LES ENFANTS DE SOCRATE. FILIATION INTELLECTUELLE ET TRANSMISSION DU SAVOIR. XVII EXXI $^{E}$ SIÈCLE, PARIS, 2008.

29. A. SCHNAPP, ANTIQUAIRES ET ARCHÉOLOGUES : RESSEMBLANCES ET DISSEMBLANCES », IN V. KRINGS, C. VALENTI (DIR.), LES ANTIQUAIRES DU MIDI, SAVOIRS ET MÉMOIRES XVIE-XIX ${ }^{E}$ SIÈCLE, PARIS, ERRANCE, 2011, P. 183-190.

\section{AUTEUR}

\section{MARIANNE ALTIT-MORVILLEZ}

Chercheur associé UMR 5608 TRACES

Université Toulouse-Jean Jaurès

marianne.altit-morvillez@neuf.fr 